\title{
L'autorégulation conjointe de la cognition et des émotions : quel impact sur les apprentissages ?
}

Bastien WAGENER, Université d'Angers, LPPL, UPRES EA 4638, France

\section{Introduction}

Aspect central de notre vie professionnelle ou personnelle, les apprentissages font partie de notre quotidien. Que ce soit au cours de formations, au travail, lors de nos loisirs ou dans nos relations aux autres, nous faisons appel à un ensemble de processus et de compétences liés par des relations complexes pour appréhender avec plus ou moins d'efficacité les problèmes que nous rencontrons. Si l'apprentissage semble complètement automatique dans certaines situations (sans réflexion consciente et intervention volontaire), dans d'autres, nous faisons appel à des stratégies bien rodées (répétition des chiffres pour retenir un numéro de téléphone par exemple) sans nous poser de questions. Parfois, il convient pourtant de s'engager dans un processus plus conscient de résolution de problème : nous nous interrogeons alors sur la meilleure stratégie à adopter pour atteindre nos objectifs et nous tâchons de réguler les processus cognitifs et émotionnels en jeu, dans les limites de nos capacités. Ces situations se présentent lorsqu'une tâche n'est ni complètement automatisée, ni intégralement nouvelle et inconnue. En effet, dans le cas d'une tâche complètement inédite nous n'avons ni stratégies d'apprentissage disponibles, ni connaissances ou expériences préalables dans le domaine : nous ne sommes alors pas en capacité d'entrer dans un processus de résolution de problème. De même, une tâche relativement aisée mais dont un paramètre va changer de manière inopinée nécessitera de faire appel à l'autorégulation de notre cognition, c'est-à-dire la métacognition (Flavell, 1987 ; Weinert).

Sur quels éléments vont s'orienter nos ressources attentionnelles volontaires lors de cette activation métacognitive ? Contrairement à ce qui est souvent admis dans la recherche, nous considérerons ici que la métacognition concerne un aspect réflexif qui a pour objet la cognition dans son sens très large de processus de traitement de l'information. Cette information n'est pas forcément exclusivement conceptuelle ou sensorielle, mais peut aussi être motivationnelle ou émotionnelle. Néanmoins, les émotions sont des «informations » possédant certaines spécificités et il nous sera nécessaire de voir en quoi leur régulation présente un cas particulier. Cependant, un clivage quasi-hermétique entre cognition et 
émotion n'a selon nous pas lieu d'être (Wagener, 2010), et certaines recherches en neuropsychologie (Damasio) montrent que ces deux dimensions sont intimement liées.

Notre objectif dans cet article est double: mieux cerner l'articulation théorique existante entre métacognition et émotions, mais aussi comprendre l'impact de ces dernières sur les apprentissages. Cet impact sera d'abord étudié sous son aspect spontané, puis surtout sous l'angle de remédiations métacognitives nouvelles dont les retombées peuvent aller audelà de la simple amélioration des performances. Notre approche se veut ainsi fondamentalement holistique tout en restant pragmatique.

\section{La métacognition : l'autorégulation de son propre fonctionnement}

La métacognition, en tant que concept regroupant l'autorégulation consciente de nos cognitions et comportements se compose de plusieurs volets que nous allons maintenant détailler. L'apprentissage autorégulé (qui peut être considéré comme un synonyme de la métacognition) est un processus actif qui implique la capacité de l'individu à appréhender une situation-problème en s'appuyant sur plusieurs compétences pour atteindre un objectif d'apprentissage spécifique (Pintrich ; Azavedo et Witherspoon; Strain, Azavedo et D’Mello) :

- la planification de son activité,

- le suivi de son activité (monitoring),

- des stratégies d'apprentissages,

- la régulation d'aspects «clés » de sa cognition, de son comportement, de sa motivation et de ses affects.

Depuis les travaux de John Flavell, la métacognition est peu à peu devenue un objet d'intérêt croissant dans le monde de l'apprentissage où l'approche exclusivement cognitive montrait ses limites. Si la métacognition peut sembler réservée à des situations d'apprentissages «classiques », elle est pourtant mise en œuvre au quotidien dans une multitude de situations de résolution de problèmes nécessitant une implication consciente et volontaire de l'individu dans ses propres activités mentales et comportements. Regroupant à la fois les connaissances qu'une personne possède sur son propre fonctionnement ainsi que les processus mêmes de suivi et de régulation volontaires de l'activité en cours (Gombert; Dunlosky et Metcalfe ; Noël), la métacognition apparaît comme un ensemble regroupant des processus conscients et leurs produits en terme de connaissances. Ainsi, ce concept parfois nommé pensée réflexive se compose d'un savoir et de compétences. Nous allons ici aborder 
séparément ces deux aspects pour des raisons discursives, mais il ne faut en aucun cas les concevoir comme indépendants et isolés des autres pans de la cognition humaine.

\subsection{Le savoir métacognitif}

Le savoir métacognitif regroupe les connaissances déclaratives et procédurales sur la cognition et sur ce qui est susceptible de l'affecter. Ces connaissances sont composées de faits, de croyances et d'épisodes verbalisables et accessibles à la conscience (Flavell, 1979). En outre, comme tout savoir, les connaissances métacognitives sont stockées en mémoire à long terme (Pintrich, Wolters et Baxter) et peuvent être récupérées suite à une recherche consciente et délibérée, ou être activées automatiquement par des indices présents dans la tâche en cours (Flavell, 1979). Ce savoir comporte plusieurs catégories : des connaissances générales sur notre fonctionnement cognitif, des connaissances sur les spécificités de différentes tâches ou types de problèmes, le savoir relatif à l'influence des contextes externes (e.g. : social, perceptif, etc.) et internes (e.g. : motivationnel, émotionnel, physiologique, etc.), ainsi que les connaissances au sujet de nos capacités cognitives (qui peuvent être évaluées les unes par rapport aux autres ou encore par rapport à celles, perçues, d'autres individus). Ces connaissances et croyances peuvent du reste être inexactes ou partiellement activées en fonction des situations et donc créer des conflits dans l'apprentissage (Noël ; Brown ; Romainville, 1993).

\subsection{Les compétences métacognitives}

Comme nous venons de l'évoquer, les compétences métacognitives se composent du suivi de l'activité cognitive et de sa régulation volontaire et consciente.

Le premier élément appelé également monitoring consiste en un suivi conscient et une évaluation de l'activité en cours. Ce suivi (parfois appelé «veille métacognitive ») permet de vérifier le déroulement des processus cognitifs et leur pertinence par rapport aux objectifs, aux expériences antérieures similaires et au plan d'action actif (et plus indirectement par rapport aux motivations). L'autoévaluation fait donc partie intégrante de ce pan de la métacognition et permet par ailleurs de détecter les erreurs. Le monitoring constitue ainsi la colonne vertébrale de la métacognition, puisque c'est sur cette base que vont se construire les connaissances métacognitives, l'ajustement des processus et stratégies ainsi que l'autocorrection. Cependant, le monitoring s'appuie sur les connaissances métacognitives pour évaluer l'activité en cours. En outre, si le monitoring est une action délibéré, les éléments sur lesquels il se base ne sont pas nécessairement tous conscients (Koriat). Enfin, cet aspect de l'autorégulation n'est lui non plus pas toujours exempt d'erreurs ou de biais (Nelson ; Koriat ; Glenberg, Wilkinson et Epstein ; Nelson, Gerler et Narens). 
La régulation métacognitive a quant à elle pour objectif de modifier les activités cognitives en cours. Cela consiste à décider de poursuivre, d'altérer ou d'arrêter certains processus cognitifs. Le contrôle implique également la planification des tâches (Brown, Bransford, Ferrara et Campione) pour atteindre un ou plusieurs objectifs fixés au préalable. Tout comme les processus cognitifs, les objectifs peuvent être réajustés au fur et à mesure, voire même totalement modifiés. Le contrôle métacognitif est souvent rapproché des fonctions exécutives et est constitué d'un ensemble complexe de compétences (Nelson et Narens ; Pintrich; Winne et Perry). Ici aussi, la mise en place d'une régulation adaptée ne peut se faire que grâce à l'activation de connaissances métacognitives, qui vont par ailleurs s'enrichir des nouvelles situations et régulations métacognitives vécues.

Les compétences métacognitives sont donc toujours contraintes (au moins partiellement) par les connaissances métacognitives disponibles. Les nouvelles expériences métacognitives et l'exercice de ces compétences vont à l'inverse enrichir et modifier les connaissances métacognitives. Ces deux aspects de la métacognition sont donc distincts mais restent en interaction permanente et s'alimentent mutuellement.

\subsection{Métacognition et apprentissages}

Intéressons-nous maintenant à l'impact de la métacognition sur les apprentissages. Premièrement, la capacité à réguler sa propre activité est fortement liée à l'habileté d'apprentissage (capacité d'acquérir de nouvelles compétences facilement) et à la capacité à résoudre des problèmes (Kruger et Dunning; Ehrlinger, Johnson, Banner, Dunning et Kruger; Brown ; Veenman). Par ailleurs, ce qui caractérise les apprenants performants est la capacité à transférer les connaissances et compétences métacognitives d'une situation à une autre (Panaoura, Philippou et Christou). Enfin, Barry J. Zimmerman avance que la compétence la plus nécessaire à un apprentissage de qualité est celle de pouvoir réfléchir sur sa propre activité d'apprentissage et de l'ajuster en fonction du contexte. Margaret Wang, Geneva D. Haertel et Herbert J. Walberg ont quant à eux montré que la métacognition est le facteur qui influence de la manière la plus efficace les apprentissages.

Plus largement, les métacognitions semblent transférables d'un domaine de la vie à un autre (Beer). Nous reviendrons dans les paragraphes suivants sur cet aspect plus intégré et transversal de la métacognition. Pour en revenir aux apprentissages, on peut considérer que cette autorégulation comporte quatre phases (Winne et Hadwin) : définition de la tâche, établissement des objectifs et planification, mise en œuvre (ou mise en actes), et enfin adaptation. Un apprenant décide donc d'abord de s'investir dans une tâche et de la décrypter, puis fixe des objectifs et des plans pour la réaliser. La mise en œuvre implique d'utiliser de 
nombreuses stratégies pour réaliser les plans élaborés précédemment, et les apprenants peuvent adapter n'importe quel aspect de leur processus d'apprentissage selon leurs expériences en cours de tâche. Ces étapes métacognitives se réalisent en fonction du contexte, des compétences disponibles et de l'évaluation de productions (mentales ou comportementales) par rapport aux normes fixées ou adoptées par l'apprenant. Cette compétence centrale qu'est l'autorégulation est donc un levier important pour améliorer les capacités d'apprentissages des individus.

Malgré notre compréhension du phénomène, il reste difficile d'améliorer les connaissances et les compétences métacognitives par le biais d'entraînements (Romainville, 2000). En effet, les remédiations proposées sont souvent limitées à des domaines très spécifiques (Nietfeld, Cao et Osborne; Delclos et Harrington) : les interventions efficaces se résument donc souvent à entraîner au suivi de son activité pour une tâche spécifique, un entraînement aux stratégies de résolution de problèmes n'étant pas suffisant seul (Romainville, 1993). Cet entraînement métacognitif peut aussi prendre la forme d'une explicitation de sa propre activité (McInerney, McInerney et Marsh ; Teong). En outre, ce type d'entraînement limité ne peut être bénéfique que pour des tâches non automatisées et présentant un niveau de difficulté certain, mais pas trop important, car l'attention portée à la métacognition ne doit pas entraver la réalisation de la tâche (Romainville, 2000). Si l'on devait proposer une remédiation plus large basée sur ces techniques de remédiation, le résultat, tout en restant encore relativement localisé, serait un programme fastidieux et lourd pour les apprenants, composé d'un ensemble d'interventions spécifiques. Cette limite est liée au fait que ces approches soient centrées sur des contenus spécifiques et pas assez sur le mode de fonctionnement (processus) que représente l'activité métacognitive. Même si cela est intéressant pour un problème ponctuel et très bien délimité, cela semble moins pertinent lorsqu'il s'agit d'intervenir à plus grande échelle. De plus, une telle approche fragmentaire d'une compétence de haut niveau, même lorsque celle-ci est, comme ici, composite, ne tient pas réellement compte de l'expérience du sujet, c'est-à-dire de la manière dont la situation est consciemment vécue, ressentie et suivie par ce dernier. Dans le cas d'une situation faisant appel à la métacognition, ce constat est primordial, comme l'avait déjà souligné Flavell dans ses travaux (1979). C'est à travers nos expériences métacognitives (ou vécu métacognitif) que nous avons la possibilité de prendre conscience de notre avancement dans une activité et de la manière dont nous ajustons notre cognition et notre comportement pour atteindre des objectifs. Nous constatons alors que la conscience et l'attention sont au centre du concept de métacognition (McGovern et Baars; Delacour). Conséquemment, il devient clair que le 
développement du savoir et des compétences métacognitives implique un aspect plus global de la personne, dans le sens où la métacognition est en lien direct avec une expérience, ou encore un vécu. Ce vécu n'implique d'ailleurs pas nécessairement une objectivité des perceptions et une justesse des évaluations métacognitives (les personnes moins performantes pour un type de tâche donné présentent un biais des évaluations métacognitives important; Ehrlinger et al. ; Kruger et Dunning ; Koriat). Avec cet aspect, nous abordons le versant phénoménologique de la métacognition, insistant par ce biais sur le fait qu'elle implique l'individu dans sa globalité. Il serait évidemment utopique de considérer que l'on pourrait dresser un portrait exhaustif individuel des différents paramètres et caractéristiques qui définissent une situation métacognitive donnée pour personnaliser les remédiations. Pourtant, lorsque l'on prône l'extrême inverse dans les remédiations cognitives (l'enseignement de «study skills », c'est-à-dire de stratégies considérées comme étant les meilleures pour un apprentissage donné quel que soit l'individu), les résultats sont assez médiocres, comme le souligne Romainville (1993). Une approche métacognitive plus éclairée que ce soit au niveau pratique ou théorique doit donc prendre en compte la manière dont les individus vivent les situations métacognitives. Ce vécu implique notamment la dimension émotionnelle, qu'il convient de définir plus précisément avant de pouvoir l'intégrer aux concepts relevant de l'autorégulation dans les apprentissages.

\section{Emotions et méta-émotions}

Nous avons vu qu'un lien relativement évident, et pourtant souvent ignoré, reliait la sphère émotionnelle à la métacognition. Souvent mise de côté dans les apprentissages comme un aspect à gérer par l'apprenant, l'émotion peut néanmoins susciter la motivation et l'entretenir, mais également favoriser la mémorisation et le rappel de connaissances (Rolls).

Il n'est pas rare d'être perturbé ou motivé par des émotions négatives ou positives lorsque l'on entreprend un travail. Même si nous ne ressentons pas en permanence d'émotions fortes, les sentiments ou «humeurs » que les émotions engendrent, créent un contexte dans lequel l'apprentissage a lieu, influençant ainsi celui-ci (de manière plus ou moins forte et bénéfique). L'émotion est ainsi une des bases de l'apprentissage (Rolls). N'importe quelle tâche peut être influencée ou modifiée par les émotions : ces dernières font même partie intégrante de nos expériences métacognitives et nous donnent des renseignements sur l'avancement de la tâche en cours et sa difficulté (Efklides). Avant d'avancer sur les interactions multiples entretenues entre émotion et métacognition, intéressons-nous à l'émotion de manière plus précise. 


\section{1. Émotion et Sentiment}

Une définition classique de l'émotion selon Klaus R. Scherer et J. Sangsue (2004) est la suivante : « réaction organisée et utile à une situation donnée ». Selon ces mêmes auteurs, le sentiment est l'expérience consciente d'une émotion et naît de l'association entre évaluation inconsciente, évaluation consciente et de verbalisation associée des composantes émotionnelles : on est donc ici dans un processus d'évaluation. On peut par ailleurs détailler cette « réaction organisée » en la séparant en plusieurs composantes. L'émotion est alors constituée de trois éléments : l'expérience subjective (sentiment), la réponse physiologique et les réactions expressives et comportementales (Hess; Kappas et Descôteaux). Selon P. Philippot, l'interaction entre cognition et physiologie est d'ailleurs nécessaire pour que naisse la facette phénoménale de l'émotion (sentiments subjectifs potentiellement conscients, c'està-dire le vécu émotionnel). Cette dépendance entre cognition et physiologie n'est pas anodine et doit être considérée comme riche et complexe. En d'autres termes, nous pouvons dire qu'en amont, l'évaluation émotionnelle d'une situation par des processus cognitifs implicites et explicites est nécessaire au déclenchement des réponses physiologiques de l'émotion. Cependant, il ne faut pas oublier qu'en aval, l'interprétation des sensations corporelles diffuses détermine en partie l'intensité et la qualité du sentiment, et donc l'évaluation de la situation contemporaine à ce sentiment.

\section{2. Émotion et Métacognition}

Les émotions sont bien entendu une part intégrante et essentielle de notre quotidien, à la base de l'émergence et du maintien de notre conscience (Damasio). Rolls insiste également sur le fait que la subjectivité des expériences conscientes et les sentiments qui les accompagnent sont nécessaires pour établir le sentiment de Soi et maintenir des buts à long terme. Il n'est donc pas étonnant que les apprentissages - et plus particulièrement la métacognition - entrent en interaction permanente avec la sphère émotionnelle, que ce soit par rapport à leur aspect conscient, ou par rapport au maintien d'objectifs. Anastasia Efklides et Chryssoula Petkaki montrent en quoi les émotions (positives ou négatives) influencent le vécu des expériences métacognitives : l'effort ressenti pendant la tâche, la satisfaction et la difficulté de la tâche sont directement liés à l'humeur dans laquelle se trouve l'apprenant au moment de la réalisation de celle-ci. De même, la confiance en ses productions et l'estimation de l'exactitude des performances (monitoring) s'effectue en fonction des connaissances métacognitives sous l'influence importante de l'humeur, des émotions et surtout des sentiments de l'individu (Wagener, Boujon et Fromage). 
En outre, la régulation de l'émotion constitue une composante essentielle $d u$ processus émotionnel. Elle implique que l'individu n'éprouve pas simplement des émotions, mais également qu'il intervienne sur l'expérience émotionnelle (de manière automatique ou volontaire), et ce grâce à des processus de suivi (monitoring) et de contrôle. Ainsi les émotions peuvent par exemple être inhibées, amplifiées, ou encore modifiées dans leur expression (Ricci Bitti). La régulation des émotions peut se situer sur le plan comportemental, expressif, ou encore sur le plan du ressenti, c'est-à-dire du sentiment. Cette régulation métacognitive des émotions est par ailleurs une composante essentielle de l'intelligence émotionnelle (Salovey et Mayer). Les émotions peuvent donc être le sujet d'un traitement métacognitif, même si elles sont bien souvent régulées de manière automatique.

Outre cette autorégulation, il existe également des émotions qui émergent vis-à-vis des émotions que nous ressentons : on parle alors de méta-émotions (Jäger et Bartsch). Celles-ci sont importantes dans la régulation émotionnelle selon Horst Mitmansgruber, Thomas N. Beck, Stefan Höfer et Gerhard Shüssler : elles constituent une des bases permettant à la métacognition des émotions de fonctionner. Elles vont en outre modifier les émotions qui les ont suscitées au fil du temps, et ce de manière automatique (Mitmansgruber et al.). Par ailleurs, les méta-émotions peuvent favoriser l'adaptation ou au contraire l'entraver, devenant alors source de troubles psychologiques lorsque cela devient récurrent (Beer). Ainsi, les méta-émotions positives favorisent l'acceptation de ses propres émotions (ce qui contribue alors au bien-être psychologique ; Neff) alors que les méta-émotions dysfonctionnelles vont au contraire favoriser l'évitement et le refus de certains aspects de la vie mentale de l'individu par lui-même (Mitmansgruber et al.). Les méta-émotions ne sont donc pas à confondre avec la métacognition: elles constituent un support supplémentaire pour la régulation métacognitive des émotions, et émergent par un processus similaire à celui décrit pour le sentiment, à ceci près qu'elles nécessitent de prêter attention à ses propres émotions (Jäger et Bartsch).

Le monitoring et la régulation des émotions constituent donc une part importante du fonctionnement émotionnel, tout en s'appuyant sur les méta-émotions associées. Les expériences émotionnelles ont également un impact à plus ou moins long terme sur les connaissances métacognitives : elles participent à la modification des théories implicites sur le monde, les autres et soi-même (Rimé). Pour résumer, nous pouvons dire que les connaissances métacognitives sont régulièrement remodelées par les expériences émotionnelles (dans une mesure plus ou moins importante à chaque fois). La mémorisation de ces expériences émotionnelles permet en outre à l'individu de se forger des outils précieux 
d'anticipation et d'adaptation, immédiatement disponibles lorsqu'un souvenir et les informations pertinentes associées sont réactivés par le contexte : l'émotion et le sentiment sont donc des activateurs de connaissances métacognitives très efficaces. L'émotion possède une influence importante et très souvent implicite sur la métacognition, et va ainsi orienter l'action. Si cela peut être très avantageux dans certains cas, cela peut aussi freiner une démarche métacognitive constructive et adaptative dans d'autres cas : la métacognition des émotions est alors un passage indispensable pour optimiser les conditions et les résultats d'un apprentissage. Eva Hudlicka précise d'ailleurs que dans une situation métacognitive, les émotions émergent en rapport avec la génération d'attentes et la fixation d'objectifs. Cela les situe comme partie intégrante du processus de décision et d'évaluation métacognitive, même si elles peuvent bien entendu être régulées et soumises à d'importantes variations d'une situation et d'un individu à l'autre. Il apparaît alors évident que les émotions, leur monitoring et leur régulation, ont également un impact sur la motivation des individus à s'impliquer ou non dans telle ou telle activité. Par conséquent, l'interrogation qui se pose est la suivante : l'individu peut-il bénéficier intentionnellement des réactions organisées suscitées par l'émotion dans une situation métacognitive? En effet, nous avons vu que les effets des émotions peuvent être autant positifs que négatifs. Ces dernières peuvent entre autres entraîner une sous-évaluation ou une surévaluation de ses performances par l'individu. Soulignons cependant que les émotions positives ont tendance à favoriser la performance et un fonctionnement métacognitif adapté et juste (Strain, Azevedo et D’Mello). Les émotions ont donc un impact important sur la sphère métacognitive, et il convient donc de ne pas les négliger. Le sentiment, comme émotion de second ordre, est nécessaire à la réalisation de l'apprentissage dans son déroulement (source des motivations, orientation des comportements) et dans sa finalité (mémorisation). Nous voyons donc ici que l'émotion et le sentiment (avec l'appui de méta-émotions) sont des facteurs en interaction avec la métacognition qui peuvent même devenir des contenus métacognitifs. Ils sont importants pour toute tâche métacognitive, dans sa genèse et sa réalisation.

\section{Les pratiques de l'attention : une perspective plus large pour intervenir sur l'autorégulation}

Après avoir exposé ce qu'était la métacognition et comment elle interagissait avec les émotions dans le cadre des apprentissages, il nous faut nous interroger sur la possibilité d'une intervention qui viserait la régulation conjointe de la cognition et des émotions afin de maximiser les effets bénéfiques sur lesdits apprentissages. Pour ce faire nous proposons de 
nous pencher sur le fonctionnent de l'autorégulation de ceux qui sont plutôt performants et épanouis dans les tâches d'apprentissage, dans le cadre de la psychologie positive. Cette dernière consiste à étudier scientifiquement les forces et les compétences des individus qui mènent leur vie au mieux et à comprendre comment ceux-ci s'y prennent (Sheldon et King). Un des éléments centraux qui permet aux personnes de se réaliser au quotidien est l'agentivité. Selon Albert Bandura, «Être un «agent» signifie faire en sorte que les choses arrivent par son action propre et de manière intentionnelle. L'agentivité englobe les capacités, les systèmes de croyance, les compétences autorégulatrices ainsi que les structures et les fonctions distribuées au travers desquelles s'exerce l'influence personnelle ; elle n'est pas une entité discrète localisable » (17). Cela rejoint la définition de l'autorégulation de Zimmerman. Cette capacité à agir intentionnellement par des actions autonomes est donc métacognitive par nature (Metcalfe et Greene) et liée au besoin de réalisation et à l'envie d'atteindre une certaine excellence dans une tâche (McClelland). C'est par l'expression de cette dimension métacognitive de manière adaptée et flexible que des émotions positives associées peuvent émerger (Beer). Selon cette perspective, nous constatons donc que la métacognition constitue une part centrale de l'épanouissement de l'individu, et qu'elle ne saurait se réaliser efficacement sans s'appuyer sur les cognitions, les capacités attentionnelles, les émotions et méta-émotions de manière consciente et intentionnelle. Une action globale permettant d'intervenir sur la métacognition, indépendamment de la tâche qui pose des difficultés aurait donc des conséquences positives de manière plus générale. Cependant, la plupart des études s'intéressant à la métacognition ignorent la dimension émotionnelle et se focalisent, nous l'avons souligné, sur une tâche bien particulière.

Rappelons ici que les différents types de remédiations efficaces se focalisent principalement sur le monitoring et l'explicitation de ses connaissances. Les résultats obtenus, certes intéressants, sont souvent limités et les méthodes ne sont pas nécessairement testées dans d'autres contextes. Il ne faut pas non plus oublier que le développement de la métacognition ne bénéficie pas à tous les types de tâches possibles (notamment selon leur degré de difficulté). Enfin, comme nous l'avons souligné dans la partie précédente, d'autres éléments peuvent favoriser ou entraver les effets bénéfiques de la métacognition, notamment les variables affectives : métacognition et motivation étant selon F. R. Weinert les deux facettes cognitives et affectives d'un même objet. C'est avec ces éléments en tête qu'il nous faut poursuivre notre investigation sur les capacités d'autorégulation. En élargissant notre perspective sur la métacognition, et étant donné son aspect holistique, il nous semble possible d'utiliser des approches globales issues du travail sur la conscience. En effet, ces approches 
sont liées aux émotions, à l'attention, à la motivation, aux pensées et à l'agentivité, tout comme la métacognition. Cette perspective de remédiations plus globales et moins situées viendrait compléter les remédiations métacognitives actuelles, ainsi que les remédiations cognitives spécifiques.

Ces méthodes, que l'on peut désigner par le terme de «pratiques de l'attention » ou PA (Varela, Thompson et Rosch ; Vaitl, Birbaumer, Gruzelier, Jamieson, Kotchoubey et al ; Cahn et Polich, 2006), regroupent les techniques de relaxation et de méditation. Celles-ci consistent à observer les contenus de la conscience et à modifier l'attention portée à ceux-ci, ce qui produit un impact sur le vécu lui-même et sur les connaissances que l'on a de son propre fonctionnement. Selon Antoine Lutz, Heleen A. Slagter, John D. Dunne et Richard J. Davidson, ces pratiques se composent de deux grandes catégories : les techniques d'attention focaliséei (focused attention) et les techniques de suivi ouvertii (open monitoring). Pour résumer, nous pouvons dire que les PA consistent en une autorégulation de l'activité physiologique et psychologique de l'individu sur la base d'une utilisation spécifique des ressources attentionnelles (Wagener, 2013). Notons par ailleurs que l'apprentissage de techniques relevant du suivi ouvert passe toujours par l'apprentissage préliminaire de techniques d'attention focalisée. Ainsi, ces pratiques de l'attention constituent littéralement un travail métacognitif puisqu'elles s'appuient sur un suivi intentionnel de sa propre expérience afin de la modifier et d'en augmenter la compréhension. Nous voyons dans les PA une piste à explorer à plusieurs points de vue. Non seulement elles permettraient d'éclaircir les mécanismes d'action de la métacognition, mais également de créer de nouvelles remédiations métacognitives plus globales. Mais quels sont les effets connus des PA ? Tout d'abord celles-ci permettent une évolution de certaines dimensions cognitives sur lesquelles s'appuie la métacognition. Plusieurs auteurs soulignent ainsi qu'une utilisation des PA pendant au moins plusieurs mois suscite une évolution très marquée de l'attention avec une allocation des ressources attentionnelles plus efficace, une augmentation de l'attention soutenue, de l'attention focalisée, de la mémoire de travail, et un meilleur monitoring (Cahn et Polich, 2006 ; Brefczynski-Lewis, Lutz, Shaefer et al. ; Chambers, Cuen Yee Lo, et Allen ; Lutz, Slagter, Dunne et Davidson ; Cahn et Polich, 2009 ; Lutz, Slagter, Rawlings et al. ; Tang et Posner). Ces changements ont évidemment des conséquences d'un point de vue neurophysiologique avec une augmentation de l'activité cérébrale frontopariétale, temporale, parahippocampale et au niveau du cortex visuel par exemple lors d'une tâche d'attention visuelle (Brefczynski-Lewis, et al.; Cahn et Polich, 2006). Le volume du cortex orbitofrontal et de l'hippocampe droits, tous deux impliqués dans la régulation émotionnelle et le 
contrôle des réponses comportementales, sont par ailleurs plus importants chez les personnes effectuant une PA (Luders, Toga, Lepore et Gazer ; Lutz et al.). Les modifications observées relèvent donc des dimensions liées à l'autorégulation. Par ailleurs, les PA engrangent des bénéfices supplémentaires : les effets positifs observés chez les personnes utilisant celles-ci sont nombreux, que ce soit au niveau de l'anxiété (Miu et al.), de la dépression (Krampen, 1999) ou du traitement de la douleur (Kanji, 2000). Cela est vrai quel que soit l'âge du sujet. Ainsi, chez l'enfant, les PA permettent de traiter la migraine (Labbé et Williamson) et de réduire les troubles comportementaux et émotionnels chez les adolescents (Goldbeck et Schimd). Chez les personnes âgées des bénéfices sont à relever au niveau des émotions, du contrôle de son propre développement, de l'autorégulation et des plaintes psychosomatiques (Krampen, 1996).

La prise de conscience de son propre fonctionnement dans les PA participe donc véritablement de la métacognition. Selon la perspective de Bishop et al., celles-ci mettent l'accent sur les composantes cognitives et émotionnelles de l'engagement conscient. L'attention autorégulée est alors focalisée sur le vécu et l'ouverture émotionnelle facilite l'acceptation et l'appréciation de toutes les expériences internes. Dans cette perspective, nous constatons que les PA impliquent la métacognition ainsi que les sphères émotionnelle et méta-émotionnelle (Snyder, Lopez et Teramoto Pedrotti).

\subsection{De nouvelles méthodes pour améliorer l'épanouissement et les apprentissages}

Nous venons de voir en quoi une approche plus globale pourrait présenter un intérêt fondamental dans le développement métacognitif des individus, tout en ayant des retombées bien plus larges que celles liées au seul apprentissage qui pose problème. Cependant, une mise en garde s'impose : il n'est pas raisonnable de penser que de telles interventions seraient une panacée qui résoudrait toute difficulté de manière rapide concernant de multiples domaines de la vie de l'individu. Bien entendu toute nouvelle approche doit être testée et ne constituera qu'un outil complémentaire aux interventions très ciblées et spécifiques liées à un domaine de compétence particulier. Cette mise en garde effectuée, nous nous proposons d'explorer ce qui pourrait constituer de nouvelles remédiations basées sur les PA.

Le lien entre PA et métacognition, nous l'avons étayé, se situe donc à plusieurs niveaux : cognitif, émotionnel et métacognitif (Wagener, 2011). Des résultats concrets concernant la métacognition ont d'ailleurs pu être observés. Dans un travail précédent (Wagener, 2013), nous avons montré - grâce à une étude de terrain chez de jeunes adultes que l'utilisation de PA chez des novices permettait d'améliorer à court terme (quatre mois environ) les connaissances et les compétences métacognitives. De la même manière, nous 
constatons que les participants formés à une PA font eux-mêmes état d'une amélioration significative de leur métacognition, de leur concentration et de la gestion du stress (Wagener, 2011). Ainsi les résultats sont à la fois objectivement et subjectivement mesurables. Il reste à explorer à quel point des performances concrètes peuvent être améliorées à moyen-terme par de telles approches. Notons qu'un aspect motivationnel est ici à prendre en compte puisque dans ces études les participants pratiquaient la technique enseignée en totale autonomie (après la phase initiale de formation). L'encadrement est donc un point essentiel qui permet d'accompagner les personnes vers une amélioration/un entraînement autonome de leur autorégulation à long terme. Une PA comme le yoga a été utilisée dans plusieurs études et impacte de manière similaire l'autorégulation, notamment à l'école (Flak et de Coulon ; Laborde). De même, la résolution de problèmes (Field, Diego et Hernandez-Reif) ainsi que des compétences recherchées dans certains métiers (précision, perception du risque, autonomie et autorégulation notamment; Marques, Ferreira, Rodrigues et Ferreira) sont améliorées grâce au yoga.

Comment pourraient alors s'organiser des remédiations métacognitives plus holistiques (RMH) ? Ce type d'intervention doit s'adresser à des petits groupes pour permettre la mise en place d'un réel échange entre les participants, tout en offrant un encadrement de qualité. En nous basant sur notre pratique et sur la formation à des PA dans d'autres contextes (Servant), il nous semble que des groupes d'environ 10 personnes favoriserait une prise en charge optimale. Un travail sur plusieurs séances serait alors envisagé. Une formation d'environ 3 mois, à raison d'une séance par semaine, permettrait l'assimilation optimale de la PA proposée. Une première séance aurait pour but d'évaluer les difficultés des participants, par le biais de questionnaires. L'adaptation d'outils existants comme des entretiens (Self-Regulated Learning Interview Schedule, Zimmerman et MartinezPons, 1986, 1988), le questionnaire de métacognitions et méta-émotions positives (PMCEQ : Beer et Moneta) ou encore un questionnaire portant sur le stress et la relaxation (Take it easy : Servant) permettraient de faire un état des lieux, à confronter aux mêmes mesures à la fin de la prise en charge. Les séances suivantes permettraient de progresser dans une PA et de proposer un échange avec les participants sur leur pratique en autonomie (basée sur un carnet de suivi qu'ils auraient à tenir entre les séances). La séance terminale permettra de repasser une évaluation armée basée sur des questionnaires, mais également d'obtenir un retour plus subjectif et qualitatif des participants. Des séances de suivi nous semblent également pertinentes pour évaluer l'évolution des participants au long cours. Cette approche devra bien entendu être adaptée en durée et en contenu en fonction de la PA retenue. À terme, il pourrait 
être intéressant d'y associer des remédiations métacognitives plus spécifiques (McInerney et al. ; Teong ; Nietfeld et al. ; Delclos et Harrington) pour traiter de problèmes très spécifiques en parallèle de l'approche globale. L'utilisation conjointe d'entretiens d'explicitation (Vermersch) pour mettre à jour les difficultés précises rencontrées par les participants constituerait une aide supplémentaire. Plusieurs entretiens seraient à envisager, et ce à plusieurs moments distincts, en parallèle de la RMH pure.

Travailler avec des populations diverses nécessitera des adaptations. Mis à part les étudiants, ces RMH pourraient être proposées à des professionnels, pour leur permettre de mieux gérer les situations conflictuelles, et de pallier certaines erreurs ou problèmes qu'ils rencontrent dans leur travail. Les remédiations métacognitives holistiques peuvent être employées auprès d'enfants et d'adolescents, à condition bien sûr de proposer des aménagements. Les séances seront dans ce cas déclinées de manière plus ludique, et le travail se fera plus progressif, avec des exercices intermédiaires. On peut néanmoins se poser la question de la pertinence de telles remédiations pour de très jeunes enfants dont la métacognition est encore à un stade précoce de développement. Ainsi, la durée des séances, les questionnaires proposés et la présentation des méthodes employées doivent s'adapter au public auquel ils sont proposés. La métacognition, utilisée au quotidien dans le milieu professionnel, personnel et les loisirs, pourra ainsi bénéficier de RMH dans des cadres variés et pour des populations différentes. Ces remédiations perdraient néanmoins de leur sens si elles n'étaient pas adaptatives : puisque le sujet est au cœur des processus de changement ciblés et engendrés dans celles-ci, son adhésion au programme est indispensable.

\section{Conclusions}

Nous l'avons vu, une approche permettant d'améliorer l'autorégulation conjointe des émotions et de la cognition est non seulement pertinente, mais également possible et prometteuse. Les effets positifs sur les activités d'apprentissage sont de plus en plus attestés, sans compter les bénéfices secondaires sur la qualité de vie que nous avons mentionnés tout au long de cet article. Des choix mieux éclairés et une autorégulation plus efficace permettent même une augmentation du bonheur chez les individus. En effet, les différences au niveau du bonheur s'expliquent essentiellement par les activités intentionnelles (Sheldon et Lyubomirsky). Celles-ci sont conscientes, requièrent un effort et sont volontairement exécutées par les individus (Snyder, Lopez et Teramoto Pedrotti). La métacognition impliquée dans ces activités et donc un moyen d'atteindre un certain épanouissement. Par ailleurs cette autorégulation est directement liée à l'optimisme et permet d'obtenir une plus 
grande satisfaction dans la vie et tout simplement plus d'épanouissement (Carver, Sheier et Weintraub ; Aykac).

Attention tout de même à ne pas croire en un remède miracle. L'approche globale ne sera premièrement pas toujours adaptée : face à un problème très circonscrit, une approche ciblée semble effectivement bien plus «rentable» en termes d'efficacité et de résultats. Cependant, nous sommes parfois confrontés à des problématiques métacognitives plus larges, et un problème spécifique peut être un révélateur d'un dysfonctionnement plus important. S'il nous faut rester très prudents et modestes quant aux effets connus du type d'approche que nous avons développé ici, cette piste nous semble pourtant pertinente et permettra de mieux cerner le fonctionnement complexe de l'individu qui autorégule intentionnellement son comportement et ses pensées. De futurs et nombreux travaux seront donc nécessaires pour adapter les PA au domaine des apprentissages et pour les rendre intelligibles pour les apprenants : sans leur adhésion au contenu de telles interventions, celles-ci resteraient bien vaines. L'approche holistique proposée ici, notamment à travers les RMH, nous semble donc très intéressante, mais nécessitera d'être mise de multiples fois à l'épreuve du terrain et de ses exigences avant de pouvoir être proposée de manière optimale à tel ou tel apprenant présentant des difficultés d'un point de vue métacognitif.

\section{Bibliographie}

Aykac, Monika. «Accentuate the Positive : Effects of Dispositional Optimism, Personality Traits and Positive Metacognitions \& Meta-emotions on Life Satisfaction ». $M M U$ Psychology Journal (2011) : 1-34.

Azevedo, R. et A. M. Witherspoon. "Self-regulated Learning with Hypermedia ». Handbook of Metacognition in Education. Dir. A. Graesser, J. Dunlosky et D. Hacker. Mahwah, NJ : Erlbaum, 2009. 319-339.

Bandura, Albert. «La théorie sociale cognitive : une perspective agentique ». Traité de psychologie de la motivation. Dir. P. Carré et F. Fenouillet. Paris : Dunod, 2009. 1545.

Beer, Nils et Giovanni B. Moneta. «Construct and Concurrent Validity of the Positive Metacognitions and Positive Meta-emotions Questionnaire ». Personality and Individual Differences 49 (2010) : 977-982.

---. « Effects of Positive Metacognitions and Meta-emotions on Coping, Stress Perception and Emotions ». Doctorate of philosophy, London Metropolitan U, London, 2011. 
Bishop, Scott. R., et al. «Mindfulness : A Proposed Operational Definition ». Clinical Psychology : Science and Practice 11.3 (2004) : 230-241.

Brefczynski-Lewis, J.A., A. Lutz, H.S. Shaefer, D.B. Levinson et R.J. Davidson. « Neural Correlates of Attentional Expertise in Long-term Meditation Practitioners ». PNAS (Proceedings of the National Academy of Sciences of the United States of America) 104. 27 (2007) : 11483-11488.

Brown, Ann L. « Metacognition, Executive Control, Self-regulation and Other More Mysterious Mechanisms ». Metacognition, Motivation and Understanding. Dir. F.R. Weinert et R.H. Kluwe. Hillsdale, NJ : Lawrence Erlbaum, 1987. 65-116.

---, Bransford, D. John, Roberta Ferrara et Joseph C. Campione. « Learning, Remembering and Understanding ». Handbook of Child Psychology : Cognitive development. Vol.3. Flavell, J.H.

Cahn, B. Rael et John Polich. «Meditation States and Traits : EEG, ERP, and Neuroimaging Studies ». Psychological Bulletin 132. 2 (2006) : 180-211.

---. « Meditation (Vipassana) and the P3a Event-related Brain Potential ». International Journal of Psychophysiology 72 (2009) : 51-60.

Carver, Charles S., Michael F. Scheier et Jagdish K Weintraub. « Assessing Coping Strategies: A Theoretically Based Approach ». Journal of Personality and Social Psychology 56 (1989) : 267-283.

Chambers, R., B. Cheun Yee Lo, et N.B. Allen. « The Impact of Intensive Mindfulness Training on Attentional Control, Cognitive Style, and Affect ». Cognitive Therapy and Research 32.3 (2008) : 303-322.

Damasio, Antonio R. Le sentiment même de soi : corps, émotions, conscience. Paris : Jacob, 1999.

Delacour, J. « An Introduction to the Biology of Consciousness ». Neuropsychologia 33.9 (1995) : 1061-1074.

Delclos, V.R. et C. Harrington. «Effects of Strategy Monitoring and Proactive Instruction on Children's Problem-solving Performance ». Journal of Educational Psychology 83.1 (1991) : 35-42.

Dunlosky, John et Janet Metcalfe. Metacognition. Thousand Oaks, CA : SAGE, 2009.

Efklides, Anastasia. «Metacognition and Affect : What Can Metacognitive Experiences Tell us about the Learning Process ? »Educational Research Review 1 (2006) : 3-14.

---, et Chryssoula Petkaki. « Effects of Mood on Students' Metacognitive Experiences ». Learning and Instruction 15 (2005) : 415-431. 
Ehrlinger, J., K. Johnson, M. Banner, D. Dunning, D. et J. Kruger. « Why the Unskilled Are Unaware : Further Explorations of (Absent) Self-insight among the Incompetent ». Organizational Behavior and Human Decision Processes 105 (2008) : 98-121.

Field, T., M. Diego et M. Hernandez-Reif. « Tai Chi/Yoga Effects on Anxiety, Heartrate, EEG and Math Computations ». Complementary Therapies in Clinical Practice 16.4 (2010) : 235-238.

Flak, Micheline et Jacques de Coulon. Des enfants qui réussissent : Le yoga dans l'éducation. Paris : Desclée De Brouwer, 1985.

Flavell, John H. « Metacognitive Aspects of Problem-solving ». The Nature of Intelligence.

Dir. L.B. Resnick, Hillsdale, NJ : Lawrence Erlbaum, 1976. 231-236.

---. « Metacognition and Cognitive Monitoring : A New Area of Cognitive-developmental Inquiry ». American Psychologist 34 (1979) : 906-911.

---. « Speculation about the Nature and Development of Metacognition ». Metacognition, Motivation, and Understanding. Dir. F. Weinert et R. Kluwe. Hillsdale, NJ : Lawrence Erlbaum, 1987. 21-29.

Glenberg, Arthur M., Alex Wilkinson et William Epstein. «The Illusion of Knowing : Failure in the Self-assessment of Comprehension ». Memory \& Cognition 10 (1982) : 597-602.

Goldbeck, L. et K. Schmid. «Effectiveness of Autogenic Relaxation Training on Children and Adolescents with Behavioural and Emotional Problems ». Journal of the American Academy of Child and Adolescent Psychiatry 42.9 (2003) : 1046-1054.

Gombert, Jean-Émile. Le développement métalinguistique. Paris : PUF, 1990.

Hess, Klaus. «Émotion ressentie et simulée ». Cognition et Émotions. Dir. Gilles Kirouac. Coimbra : Imprensa da Universidade, 2004. 115-128.

Hudlicka, Eva. « Modeling Interaction Between Metacognition and Emotion in a Cognitive Architecture ». Proceedings of the AAAI Spring Symposium on Metacognition in Computation, AAAI Technical Report SS-05-04, Menlo Park, CA : AAAI P, 2005. 5561.

Jäger, Christoph et Anne Bartsch. « Meta-Emotions ». Grazer Philosophische Studient 73 (2006) : 179-204.

Kanji, Nasim. "Management of Pain through Autogenic Training ». Complementary Therapies in Nursing \& Midwifery 6 (2000) : 143-148. 
Kappas, A. et J. Descôteaux. "Les promesses et limites de l'étude de l'émotion en laboratoire ». Cognition et Émotions. Dir. Gilles Kirouac. Coimbra : Imprensa da Universidade, 2004. 11-36.

Koriat, Asher. «Monitoring One's Own Knowledge during Study : A Cue-utilization Approach to Judgments of Learning ». Journal of Experimental Psychology : General 126 (1997) : 349-370.

---. «Metacognition and Consciousness ». Cambridge handbook of consciousness. Dir. Philip David Zelazo, Morris Moscovitch et Evan Thompson. New York : Cambridge UP, 2007. 289-326.

Krampen, Günter. « Evaluation of the Effectiveness of Autogenic Training in Gerontopsychology ». European Psychologist 1.4 (1996) : 243-254.

---. « Long-term Evaluation of the Effectiveness of Additional Autogenic Training in the Psychotherapy of Depressive Disorders ». European Psychologist 4.1 (1999) : 11-18.

Kruger, Justin et David Dunning. «Unskilled and Unaware of It : How Difficulties in Recognizing One's Own Incompetence Lead to Inflated Self-Assessments ». Journal of Personality and Social Psychology 77.6 (1999) : 1121-1134.

Labbé, Élise L. et Donald A. Williamson. « Treatment of Childhood Migraine Using Autogenic Feedback Training ». Journal of Consulting and Clinical Psychology 52.6 (1984) : 968-976.

Laborde, Magalie. «La pratique du yoga à l'école primaire : pour une co-disponibilité ». Mémoire professionnel en pédagogie. IUFM de l'Académie de Montpellier, Perpignan, France, 2006.

Luders, Eileen, Arthur W. Toga, Natasha Lepore et Christian Gazer. « The Underlying Anatomical Correlates of Long-term Meditation : Larger Hippocampal and Frontal Volumes of Gray Matter ». NeuroImage 45 (2009) : 672-678.

Lutz, Antoine, Heleen A. Slagter, John D. Dunne et Richard J. Davidson. « Attention Regulation and Monitoring in Meditation ». Trends in Cognitive Sciences 12.4 (2008) : 163-169.

---, Heleen A. Slagter, N.B. Rawlings, A.D. Francis et L.L. Greischar. « Mental Training Enhances Attentional Stability : Neural and Behavioral Evidence ». The Journal of Neuroscience 29.42 (2009) : 13418-13427.

Marques, Carla Susana, João Ferreira, Ricardo Gouveia Rodrigues et Mariza Ferreira. «The Contribution of Yoga to the Entrepreneurial Potential of University Students : A SEM 
Approach ». International Entrepreneurship and Management Journal 7.2 (2010) : 255-278.

McClelland, David C. Human Motivation. Glenview, IL : Scott \& Forseman, 1985.

McGovern, K. et B.J. Baars. « Cognitive Theories of Consciousness ». Cambridge Handbook of Consciousness. Philip David Zelazo, Morris Moscovitch et Evan Thompson. New York : Cambridge UP, 2007. 177-205.

McInerney, Valentina, Dennis M. McInerney et Herbert W. Marsh. « Effects of Metacognitive Strategy Training Within a Cooperative Group Learning Context on Computer Achievement and Anxiety : An Aptitude-Treatment Interaction Study ». Journal of Educational Psychology 89.4 (1997) : 686-695.

Metcalfe, Janet et Matthew Jason Greene. " Metacognition of Agency ». Journal of Experimental Psychology : General 136.2 (2007) : 184-199.

Mitmansgruber, Horst, Thomas N. Beck, Stefan Höfer et Gerhard Schüssler. «When You Don't Like what you Feel : Experiential Avoidance, Mindfulness and Meta-emotion in Emotion Regulation ». Personality and Individual Differences 46.4 (2009) : 448453.

Miu, Andrei C., Renata M. Heilman et Mircea Miclea. «Reduced Heart Rate Variability and Vagal Tone in Anxiety : Trait versus State, and the Effects of Autogenic Training ». Autonomic Neuroscience : Basic and Clinical 145 (2009) : 99-103.

Murakami, Masato, Kazuyoshi Koiki, Mitsumi Ashihara, Toshio Matsuno, Mami Tazoe et Katsura Taisaku. «Recent Advance of Autogenic Training in Clinical Practice of Psychosomatic Medicine in Japan ». International Congress Series 1287 (2006) : 240245.

Neff, Kristin D. «The Development and Validation of a Scale to Measure Self-compassion ». Self and Identity 2.3 (2003) : 223-250.

Nelson, Thomas O. «Consciousness and Metacognition ». American Psychologist 51 (1996) : 102-116.

---, Daniel Gerler et Louis Narens. « Accuracy of Feeling-of-Knowing Judgments for Predicting Perceptual Identification and Relearning ». Journal of Experimental Psychology : General 113 (1984) : 282-300.

---. « Metamemory : A theoretical Framework and New Findings ». The Psychology of Learning and Motivation 26 (1990) : 125-141. 
Nietfeld, John L., Li Cao et Jason W. Osborne. « The Effect of Distributed Monitoring Exercises and Feedback on Performance, Monitoring Accuracy, and Self-efficacy ». Metacognition Learning 1 (2006) : 159-179.

Noël, Bernadette. La métacognition. Paris : De Boeck U, 1997.

Panaoura, Areti, George Philippou et Constantinos Christou. «Young Pupils’ Metacognitive Ability in Mathematics ». Building Structures in Mathematical Knowledge. Proceedings of the Third Conference of the European Society for Research in Mathematics Education. Mariotti, M.A. 2003.

Philippot, P. « Facteurs cognitifs et réactions corporelles dans le processus émotionnel ». Dir. Gilles Kirouac Coimbra : Imprensa da Universidade, 2004. 37-56.

Pintrich, Paul R. « The Role of Goal Orientation in Self-regulated Learning ». Handbook of Self-Regulation. Dir. Monique Boekaerts, Paul R. Pintrich et Moshe Zeidner. San Diego, CA : Academic P, 2000. 451-502.

---, Wolters, Christopher A. et Gail P. Baxter. « Assessing Metacognition and Self-regulated Learning ». Issues in the Measurement of Metacognition. Dir. Gregory Schraw et James C. Impara. Lincoln, NE : Buros Institute of Mental Measurements, 2000. 4397.

Ricci Bitti, Pio E. «La régulation des comportements expressifs émotionnels ». Cognition et émotions. Dir. Gilles Kirouac. Coimbra : Imprensa da Universidade, 2004. 157-170.

Rimé, B. «Émotion et mémoire : La rémanence des expériences émotionnelles ». Cognition et émotions. Dir. Gilles Kirouac. Coimbra : Imprensa da Universidade, 2004. 79-96.

Rolls, E.T. «Emotion, Higher Order Syntactic Thoughts, and Consciousness ». Frontiers of Consciousness. Dir. L. Weiskrantz et M.K. Davies. Oxford : Oxford UP, 2007. 131167.

Romainville, Marc. Savoir parler de ses méthodes. Bruxelles : De Boeck U, 1993.

---. « Savoir comment apprendre suffit-il à mieux apprendre ? Métacognition et amélioration des performances ». Pour une pensée réflexive en éducation. Dir. Richard Pallascio et L. Lafortune. Sainte-Foy : PU du Québec, 2000. 71-86.

Salovey, Peter et John D. Mayer. «Emotional Intelligence ». Imagination, Cognition, \& Personality 9.3 (1990) : 185-211.

Scherer, Klaus R. et J. Sangsue. « Le système mental en tant que composant de l'émotion ». Cognition et émotions. Dir. Gilles Kirouac. Coimbra : Imprensa da Universidade, 2004. 11-36. 
Servant, Dominique. La relaxation : nouvelles approches, nouvelles pratiques. Elsevier Massons, 2009.

Sheldon, Kennon M. et Laura King. «Why Positive Psychology Is Necessary. "The American Psychologist 56.3 (2001) : 216-217.

---, et Sonja Lyubomirsky. « Achieving Sustainable Next Happiness : Prospects, Practices, and Prescriptions ». Positive Psychology in Practice. Dir. P.A. Linley et S. Joseph. Hoboken, NJ : Wiley, 2004. 127-145.

Snyder, C. R., Shane J. Lopez et Jennifer Teramoto Pedrotti. Positive Psychology. Thousand Oaks, CA : SAGE, 2011.

Stetter, Friedhelm et Sirko Kupper. «Autogenic Training : A Meta-analysis of Clinical Outcome Studies ». Applied Psychophysiology and Biofeedback 27.1 (2002) : 45-98.

Strain, Amber Chauncey, Roger Azevedo et Sidney K. D’Mello. « Using a False Biofeedback Methodology to Explore Relationships between Learners' Affect, Metacognition, and Performance ». Contemporary Educational Psychology 38 (2013) : 22-39.

Tang, Yi-Yuan et Michael I. Posner. « Attention Training and Attention State Training ». Trends in Cognitive Sciences 13.5 (2009) : 222-227.

Teong, S.K. «The Effect of Metacognitive Training on Mathematical Word-problem Solving ». Journal of Computer Assisted Learning 19 (2003) : 46-55.

Vaitl, Dieter, Niels Birbaumer, John Gruzelier, Graham A. Jamieson, Boris Kotchoubey et al. «Psychobiology of Altered States of Consciousness ». Psychological Bulletin 131 (2005) : 98-127.

Varela, Francisco, Evan Thompson et Eleanor Rosch. L'inscription corporelle de l'esprit. Paris : Seuil, 1993.

Veenman, Marcel V.J. «Giftedness : Predicting the Speed of Expertise Acquisition by Intellectual Ability and Metacognitive Skillfulness of Novices. Meta-Cognition : A Recent Review of Research, Theory and Perspectives. Dir. M.F. Shaughnessy, Marcel V.J. Veenman et C. Kleyn-Kennedy. New York : Nova Science, 2008. 207-220.

Vermersch, Pierre. L'entretien d'explicitation. Issy-les-Moulineaux: ESF, 2006.

Wagener, Bastien. « Développement et transmission de la métacognition ». Thèse de doctorat en psychologie, U d'Angers, France, 2011.

---. « Autogenic Training, Metacognition and Higher Education ». Educational Psychology 33.7 (2013) : 849-861.

---, Christophe Boujon et Benoît Fromage. «Métacognitions, émotions et motivations ». Psychologie internationale, pratiques et recherche 1, 2010 : 89-109. 
Wallon, Henri. L'évolution psychologique de l'enfant. Paris : Colin, 2011 (1941).

Wang, Margaret C., Geneva D. Haertel et Herbert J. Walberg. « What Influences Learning ? A Content Analysis of Review Literature ». Journal of Educational Research 84.1 (1990) : 30-43.

Weinert, Franz E. « Introduction and Overview : Métacognition and Motivation as Determinants of Effective Learning and Understanding ». Metacognition, Motivation and Understanding. Dir. F.R. Weinert et R.H. Kluwe. Hillsdale, NJ : Lawrence Erlbaum, 1987. 1-16.

Winne, Philip H. et A.F. Hadwin. «Studying as Self-regulated Learning ». Metacognition in Educational Theory and Practice. Dir. Douglas J. Hacker, John Dunlosky et Arthur C. Graesser. Hillsdale, NJ : Erlbaum, 1998. 277-304.

---, et Nancy E. « Measuring Self-regulated Learning ». Handbook of Self-Regulation. Dir. Monique Boekaerts, Paul R. Pintrich et Moshe Zeidner. San Diego, CA : Academic P, 2000. 531-566.

Zimmerman, Barry J. « Self-regulatory Cycles of Learning ». Conceptions of Self-Directed Learning. Starka / Münster : Waxmann, 2000. 221-234.

---, et Manuel Martinez-Pons. « Development of a Structured Interview for Assessing Student Use of Self-regulated Learning Strategies ». American Educational Research Journal 23 (1986) : 614-628.

---. « Construct Validation of a Strategy Model of Student Self-regulated Learning ». Journal of Educational Psychology 80.3 (1988) : 284-290.

\section{NOTES}

Les techniques d'attention focalisée (focused attention) consistent en la concentration mentale de l'individu sur un objet réel, une image mentale ou une partie du corps pendant une certaine durée. Ces techniques permettent de développer les capacités attentionnelles et d'autorégulation du sujet.

Les techniques de suivi ouvert (open monitoring) sont abordées après l'apprentissage des techniques d'attention focalisée. Ici, il s'agit pour l'individu de prendre conscience de l'enchaînement de ses expériences conscientes sans prêter plus d'attention à l'une d'entre elles en particulier. L'objectif est notamment d'augmenter la compréhension que l'individu a de son propre fonctionnement et de développer ses capacités de suivi conscient de ses propres cognitions, émotions et actions. 